\title{
LA UTILIZACIÓN DE TABLETS ENTRE PREADOLESCENTES. LOS CASOS DE ZARAGOZA (ESPAÑA) Y PAU (FRANCIA)
}

\author{
Dr. Rubén Ramos Antón ${ }^{1}$ \\ Universidad de Castilla-La Mancha, Cuenca, España \\ ruben.ramos@uclm.es \\ ORCID iD: https://orcid.org/0000-0001-7023-6463 \\ Dr. Jocelyn Lachance \\ Univeristé de Pau et des Pays de l'Adour, Pau, Francia \\ jocelyn.lachance@univ-pau.fr \\ ORCID iD: https://orcid.org/0000-0003-3460-9722
}

Recibido el 30 de septiembre de 2019

Aceptado el 5 de mayo de 2020

\section{Resumen}

Esta investigación analiza la forma en la que los preadolescentes de Zaragoza (España) y Pau (Francia) utilizan las tablets, como primer dispositivo que hacen propio en el mundo digital y la forma en la que este influye en su socialización con sus pares. A través de una metodología cualitativa (entrevistas en profundidad) se investigó a 22 jóvenes de una y otra ciudad. Los resultados muestran que, a pesar de las diferencias culturales entre ambos países y ciudades, los preadolescentes manifiestan una forma muy similar de relacionarse y utilizar las tablets, siendo el nivel de penetración de esta tecnología parejo en todos los casos investigados. No obstante, sí se aprecian importantes diferencias a la hora de interpretar los espacios de ocio en los que se encuentran con sus amistades.

Palabras clave: Alfabetización tecnológica, preadolescentes, tablets, tecnologías, uso de Internet, competencia mediática.

\footnotetext{
1 Este autor recibe financiación del Fondo Europeo de Desarrollo Regional (FEDER) convocatoria 2018/11744
} 


\title{
THE USE OF TABLETS IN PRETEENS. THE CASES OF ZARAGOZA (SPAIN) AND PAU (FRANCE)
}

\begin{abstract}
This research analyzes the way in which preteens (tweens) in the cities of Zaragoza (Spain) and Pau (France) use tablets, as the first device they make their own in the digital world and how it influences their socialization with their peers. To get to that goal, through a qualitative methodology (in-depth interviews) a total of 22 young people from both cities were investigated. The results show that despite the cultural differences between both countries and cities, the preteens of Zaragoza and Pau express a very similar way to relate and use the tablets, being the level of penetration of this technology very similar in all the cases investigated. However, there are important differences when interpreting the leisure spaces in which they meet their friends.
\end{abstract}

Keywords: Technological literacy; preteens; Tablets; technologies; Internet use; media literacy. 
Introducción

A

pesar de que su implantación en los últimos años ha pasado por una clara desaceleración en el número de ventas (Ditendria, 2018: 3; Fundación Telefónica, 2018: 109), las tablets continúan siendo un elemento tecnológico clave en el actual desarrollo de la era digital, especialmente para algunos usos. De hecho, en España durante el año 2017 la tasa de penetración tecnológica de este dispositivo alcanzó el $74 \%$ y el tiempo que se le dedicó diariamente era de 1 hora 37 minutos (Ditendria, 2018: 14). En Francia, un estudio realizado por el Observatorio de Orange mostró que en 2012 más del 70\% de los niños menores de 12 años usaban una tablet en casa. En 2014, el 32,6\% de los hogares estaban equipados con el dispositivo, el 10,3\% de los mayores de 13 años tenían contacto diario con una tablet y el $86,5 \%$ de estos contactos se hicieron en casa. En 2015, el 47\% de los adolescentes franceses de entre 12 y 17 años tenían un touch pad, mientras que en 2011 esta tasa era solo del 7\% (Credoc, 2016). En 2017, la encuesta anual "Junior connect" de Ipsos reveló que el $36 \%$ de los niños de 7 a 12 años tenían su propia tablet y que más de la mitad de los hogares con al menos un niño disponía al menos de una de ellas (INSEE, 2017).

Aparte de su componente de ocio y lúdico, las tablets se incorporaron hace años como elemento de apoyo en la educación reglada, incluso en sus primeras etapas. Diversos estudios han analizado la introducción y el alcance de este dispositivo tecnológico como soporte para el aprendizaje en diferentes países (Asorey y Gil, 2009; Cáceres, Hinojo y Aznar 2011; Nogueira y Ceinos, 2015; Soykan, 2015; O'Connor y Jackson, 2016; Neumann, 2017; Reina, Pérez y Quero, 2017; Hove, Vanderhoven y Cornillie, 2017; Myrtil et al., 2018; Zhu et al., 2018). El propio Bill Gates reconoció en 2008 la iniciativa del Gobierno de Aragón por introducirlas en las aulas ${ }^{2}$, que en este caso jugaron un papel importante en áreas rurales, tradicionalmente apartadas de los avances tecnológicos. Las posibilidades que los dispositivos abren en el mundo de la educación son diversas, también a través de la utilización de los juegos (Miller y Kocurek, 2017). Estudios más recientes también ponen de manifiesto hasta qué punto las familias asumen la relación que existe entre las tablets y la educación reglada (Sánchez y Ricoy, 2018). Si bien, tal y como muestra esta investigación, el uso de estas herramientas no se encuentra totalmente normalizado, ya que existen diversas interpretaciones sobre si el dispositivo resulta útil. La opinión de los padres y madres se encuentra dividida ante la posibilidad de que la utilización de las tablets suponga un apoyo para la obtención de resultados académicos.

2 "Microsoft persigue crear nuevas oportunidades para todas las personas a través de la Educación". Nota de prensa de Microsoft del 23 de enero de 2008. Recuperado de https://goo.gl/tW6qgD 
Discusiones similares se producen prácticamente en todo el mundo. Pham y Lim (2019) analizan, por ejemplo, el papel que desempeñan las tablets entre los preescolares de Vietnam. En ese país se identifica por parte de los padres (especialmente las madres) el dispositivo como una útil herramienta educativa capaz de dar ventaja a los niños que se familiaricen con su uso.

La integración de los dispositivos tecnológicos en el aula (principalmente tablets y smartphones) no se encuentra resuelta ni siquiera en los más altos niveles formativos (Figueras-Maz, Masanet y Ferrés, 2017). No obstante, la presencia de estos elementos en las clases resulta una evidencia, que puede variar dependiendo de cuestiones sociales, culturales o económicas, como se demuestra en el estudio de Shuter, Cheong y Chen (2016) entre universitarios norteamericanos y daneses.

En Estados Unidos, Connell, Lauricella, y Wartella (2015) establecen una relación clara entre la familiaridad con los dispositivos digitales por parte de los padres y el uso que de ellos hacen los hijos, además de encontrar condicionantes (género, edad, etnia o grado de formación) que determinan el uso en común. En Bélgica, Beyens y Beullens (2017) identifican las tablets como foco de conflictos familiares. Los niños que menos tiempo pasan con ellas protagonizan más problemas con sus padres, mientras que aquellos que ven más restringido su uso tienen más problemas con sus progenitores por este motivo. Idénticos problemas generados por el tiempo prestado a tablets y teléfonos móviles se descubren en Estados Unidos con familias con niños menores de ocho años (Wartella et al., 2014).

Las tablets se encuentran, sin embargo, muy lejos del nivel de penetración que han alcanzado los teléfonos móviles, especialmente los smartphones, que en muy poco tiempo lograron convertirse en el elemento tecnológico que más rápidamente se había extendido en la historia (Pisani y Piotet, 2009: 277). En 2018 se estimaba que había en el mundo 107 líneas de telefonía móvil por cada cien habitantes, con una penetración que oscila por regiones entre las 76 líneas por cada 100 habitantes en África y las 136,8 en el espacio de la antigua Unión Soviética (UIT en ONTSI, 2019: 29). A lo largo de 2017 el número de líneas de telefonía móvil ya habían superado al total de la población mundial (Fundación Telefónica, 2018: 95).

Los smartphones ocupan claramente una posición central entre los dispositivos electrónicos, convirtiéndose asimismo en la principal vía de acceso a Internet. En 2016 en España el 91,7\% de los internautas se conectaban a la Red a través de sus teléfonos inteligentes (Fundación Telefónica, 2017: 110).

Del mismo modo, el acceso a la posesión de estos dispositivos se generaliza entre edades cada vez más precoces. En España el 69,1\% de los jóvenes entre 10 y 15 años disponía de un teléfono inteligente en 2017 (INE, 2017). El smartphone es visto como un sinónimo de mayor libertad para los adolescentes (Lachance, 2016), al mismo tiempo que se convierte en un objeto frecuentemente obtenido por parte de algunos niños y niñas como consecuencia de ritos de paso, como podría ser la 
comunión católica, del mismo modo en el que, en el pasado, se obtenía el primer reloj, que convertía simbólicamente al individuo en un ser adulto (Feixa, 2014: 108). Asimismo, el smartphone también es considerado como un instrumento para la gestión del tiempo para los adolescentes (Lachance, 2011).

Ante esta situación de predominio de la telefonía móvil y de cada vez mayor precocidad a la hora de adquirir los teléfonos, los otros dispositivos (ordenadores y tablets principalmente) desempeñan diferentes usos y funciones, que alcanzan un papel relevante especialmente entre ciertos colectivos. En Estados Unidos algunos estudios (Lauricella et al., 2014; Rideout, 2015; 2016) muestran que las tablets son más populares entre los preadolescentes, mientras que la posesión de smartphones se hace más presente conforme los niños van creciendo. Las actividades que prefieren desarrollar en ellas son juegos, vídeos, escuchar música, navegar por Internet o participar en redes sociales, entre otras (Rideout, 2015: 58). Actividades que, como se viene observando desde hace años, convergen en diferentes formas de consumir los productos audiovisuales y que se intensifican conforme aumenta el consumo de los diferentes dispositivos (Duursma, Meijer y De-Bot, 2017).

En su investigación sobre estudiantes universitarios de Estados Unidos ChanOlmsted y Shay (2016) revelan que la posesión de tablets en este colectivo resulta complementaria a la telefonía móvil, adquiriendo un significado diferente como sustitución de otros medios, como la televisión.

Por otro lado, la convivencia o incluso complementariedad de las tablets con la televisión resulta patente en algunas investigaciones. En Estados Unidos McCreery y Krugman (2015), así como D'heer y Courtois (2014) en Bélgica, señalaron que los dispositivos electrónicos móviles, como las tablets, se encontraban plenamente integrados a la hora de consumir televisión. En la misma línea se sitúa la encuesta realizada por Gil y Liu (2017), en la que un $75 \%$ de las personas encuestadas en veinte países manifiesta practicar la "doble pantalla" cuando asisten a programas de televisión relacionados con la política. La casuísitica resulta diferente en cada contexto, teniendo en cuenta la situación política y de libertades públicas de cada uno de ellos.

Como señala Dias (2016) las motivaciones para la realización de estas prácticas son variadas y en parte, sobre todo entre los más jóvenes, se identifica una necesidad de estar constantemente conectado (instantaneity addiction).

El efecto que estos dispositivos ha provocado en la forma en la que se consumen tanto las noticias como otros medios, como es el caso de la televisión, también ha sido objeto de numerosos estudios en los últimos años. (Chyi y Chadha, 2012; Gershon, 2013; Wei, 2013; Madison, 2015; Morris y Patterson, 2015; Cauwenberge, D'Haenens y Beentjes, 2015; Lomborg y Mortensen, 2017; Dunaway et al., 2018; Neijens y Voorveld, 2018). Los cambios también resultan presentes en las prácticas de los profesionales de los medios de comunicación (Mackay e Ivory, 2014). 
Las tablets resultan, por lo tanto, un elemento sustancial del actual desarrollo de las tecnologías de la información, muy presentes en los hogares de todo el mundo. Sin embargo, al ocupar una función no central (que claramente desempeñan los teléfonos móviles) es habitual su uso para funciones más concretas, bien en la educación, bien en el ocio, con frecuencia relacionado con la práctica de la doble pantalla.

\section{Zaragoza (Aragón) y Pau (Bearne). La penetración tecnológica en dos territorios periféricos}

A ambos lados de los Pirineos tanto Aragón como el Bearne han desempeñado roles periféricos en sus respectivos estados. Los dos territorios se han situado históricamente lejos de los centros de poder político y económico de España y Francia. Los avances técnicos, el progreso, llegaron a ambos lados de los Pirineos principalmente en otros lugares mejor situados política o económicamente (Madrid, Barcelona, París, Marsella, etc.). En el caso de Aragón, la actual comunidad autónoma comenzó el siglo XX siendo un territorio eminentemente rural, en el que la población activa vinculada al sector primario se situaba muy por encima de la media española (Fernández, 1997: 52). Actualmente, la economía aragonesa representa el 3,1\% del Valor Agregado Bruto estatal, con una avanzada tercerización y con un peso de la industria que ha mejorado incluso su escaso peso en el conjunto estatal (Germán, 2012: 335). A lo largo de los años este peso de la economía aragonesa en el conjunto de España se mantiene en la misma proporción $(3,1 \%)(I N E, 2019 a)$. Si bien algunos indicadores, como es el caso del PIB per cápita, se sitúan por encima de la media estatal, este se ve muy condicionado por la baja densidad de población de la comunidad. Según datos de 2019 (INE, 2019b) Aragón cuenta con una densidad de población de 27,6 habitantes por kilómetro cuadrado, muy lejos de la media estatal, que se situaría en 92,9.

En cuanto al Bearne, en 2016 el PIB per cápita de Aquitania todavía era inferior a la media francesa (27.000 euros frente a 31.800) y permanecía muy alejado al de zonas como îlle de France (51.100 euros). En el caso aragonés el PIB per cápita se situaba en 29.000 euros, por encima de la media española (23.800) pero sensiblemente por debajo de Madrid (36.400) (ver Anexo 1).

Las dos principales ciudades de ambos territorios, Zaragoza y Pau, cuentan con estrechos vínculos históricos, económicos y culturales con profundas raíces. Pau ejerce de capital del departamento de Pyrénées-Atlantiques (dentro de la actual región de Nouvelle Aquitaine), y es la principal ciudad del histórico Bearne. Por su parte, Zaragoza, ciudad más poblada del eje central del Ebro, desempeña el papel de capital de la comunidad autónoma de Aragón.

Ambas se sitúan a una distancia de menos de 300 kilómetros por carretera y se encuentran hermanadas oficialmente desde 1960, momento en el que todavía se 
encontraban unidas directamente por ferrocarril, a través del paso transfronterizo de Canfranc.

Los datos tanto macroeconómicos, como de nivel de vida de ambas regiones registran unos parámetros muy similares ${ }^{3}$, incluida la penetración tecnológica en lo relativo al acceso habitual a Internet de sus ciudadanos. Posiblemente, las variables en las que las diferencias son mayores son las relativas al número de personas que nunca usaron un ordenador. En 2015 (último año del que se dispone de datos, un $16 \%$ de la población aragonesa nunca había utilizado un ordenador, mientras que en Aquitania este porcentaje descendía hasta la mitad, un 8\%) (ver Anexo 2).

No obstante, sigue habiendo cierta diferencia con los centros de los dos respectivos estados (España y Francia) que en ambos casos (Comunidad de Madrid e îlle de France) registran parámetros mucho mayores. Resulta por este motivo interesante recordar que tanto Aragón como Aquitania han ocupado tradicionalmente un lugar periférico en sus respectivos estados, lo que todavía se traslada a algunos de los parámetros analizados.

Los datos actuales, sin embargo, dan una muestra del acercamiento de los niveles de vida, especialmente en los indicadores que tienen más relación con los elementos tecnológicos que caracterizan a la sociedad de la información, a ambos lados del Pirineo Central.

Es precisamente una característica del actual desarrollo de las tecnologías de la información la relativización o redefinición de la distancia física. A ella se han referido numerosos estudios desde que McLuhan (1996: 110) definiese la extensión del poder sobre una superficie cada vez más homogénea o Debord (2005: 144) hablase de supresión de la distancia geográfica. Otros autores que han analizado la revolución tecnológica experimentada en los últimos años también han analizado un proceso por el que, a través de las actuales tecnologías de la información, la distancia física deja de tener el sentido que se le atribuyó en el pasado (Fidler, 1998: 159; Manovich, 2005: 232; Feixa, 2014:115). Por su parte Lévy (2004) habla de "distancia cero" o Doc Searls (Pisani y Piotet, 2009: 248) del "cero gigante" que coloca a todas las personas a la misma distancia de todos los lugares.

De este modo, centro y periferia ya no representan posiciones geográficas lejanas (Ramos, 2016), mezclándose en el día a día, lo que se traduce en el caso de

\footnotetext{
${ }^{3}$ Eurostat todavía no contempla como unidad administrativa la región de Nouvelle Aquitaine, por lo que se manejan datos de la región de Aquitaine, a la que pertenecía Pau hasta la aprobación de la ley N²015-29 du 16 janvier 2015 relative à la délimitation des régions, aux élections régionales et départementales et modifiant le calendrier electoral. (Ley N²015-29 del 16 de enero de 2015 relativa a la delimitación de las regiones, a las elecciones generales y departamentales y modificación del calendario electoral).
} 
Zaragoza y Pau (Aragón y Bearne) a nivel de penetración y acceso a las tecnologías de la comunicación.

\section{Jóvenes, espacios y tecnología}

Boyd (2008) se aproxima al modo en la que los jóvenes se relacionan con la tecnología como una forma de continuar su proceso de socialización, en el que su identidad personal es parcialmente definida por ellos mismos y parcialmente por sus pares. Se trata de una oportunidad para desarrollar las relaciones sociales de los jóvenes lejos de la mirada de los adultos que se lleva observando desde hace años (Fluckiger, 2006). Las tecnologías de la información, por lo tanto, forman parte de la construcción de la persona y de la socialización, desde sus edades más tempranas y se convierten en una herramienta para la construcción del yo en un mundo hiperconectado.

Las tecnologías de la información suponen una forma de evadirse del control parental y de encontrarse con sus pares. Del mismo modo que en algunas sociedades la búsqueda de la construcción de la identidad se cimentaba a través de la llamada cultura de la habitación (Livingstone, 2007; Glévarec, 2010), un concepto que proviene de los inicios del movimiento feminista (Woolf en Feixa, 2014: 130) y que, a partir de la segunda mitad del siglo XX, comenzó a relacionarse con la construcción de la identidad de los jóvenes. Esa construcción de la identidad, que responde a la necesidad de crear un mundo personal (Hernández, Yáñez y Carrera, 2017), evoluciona y se adapta a las nuevas formas de comunicación, como pueda ser en los últimos años el uso de los selfies o de la fotografía digital (Schwarz, 2009; Lachance, 2013; Willem, Tortajada y Figueras-Maz, 2017) o del WhatsApp (Vidales-Bolaños y Sádaba-Chalezquer, 2017). Esta última continúa año tras año siendo una de las principales aplicaciones de los smartphones, como lo demuestra el hecho de que en los últimos tres años el tráfico de este tipo de mensajería instantánea se multiplicó por ocho (Fundación Telefónica, 2018: 75). En definitiva, los niños y jóvenes utilizan este tipo de tecnologías para sus propios intereses (Danby et al., 2013), entre los que se encuentran de un modo destacado construir sus personalidades y relacionarse con sus pares.

El aprendizaje y la socialización, por otro lado, también se ha desarrollado tradicionalmente en la calle, un lugar en el que se suceden las primeras experimentaciones de la vida, "la alfabetización de la calle" a la que se refiere Cahill (2000). El espacio público puede adquirir diferentes significaciones para los jóvenes y los adolescentes en función de las experiencias que estos experimentan en él (Ortiz, Prats y Baylina, 2014).

Teniendo en cuenta, por lo tanto, la importancia de los espacios públicos para la construcción de la personalidad y la socialización de los individuos, así como el papel que desempeñan las tecnologías de la comunicación en ese mismo proceso socializador entre los jóvenes, nos proponemos conocer la forma en la que 
experimentan su relación con las tecnologías de la comunicación a ambos lados del Pirineo. En concreto, se analizan las similitudes y diferencias entre preadolescentes de Zaragoza y Pau, como principales ciudades de Aragón y Bearne, dos territorios vecinos de los Pirineos Centrales.

Las hipótesis principales que se plantean en la investigación son las siguientes:

Hipótesis 1: Las tablets juegan un importante papel en la vida de los preadolescentes, especialmente antes de que estos adquieran su primer teléfono móvil. Representan el primer dispositivo con el que los jóvenes experimentan en el mundo digital.

Hipótesis 2: El grado de penetración tecnológica y la forma en la que esta se ha interiorizado por todo el mundo han provocado que estas circunstancias se den de forma similar en Aragón y Bearne.

Hipótesis 3: El espacio público sigue siendo el lugar preferido por los preadolescentes para encontrarse con sus pares, a pesar de que también utilicen dispositivos tecnológicos para ello.

\section{Material y metodología}

La presente investigación forma parte de un estudio más extenso en el que se analizan los hábitos culturales y tecnológicos de jóvenes de ambos lados de los Pirineos Centrales. Se parte de una metodología cualitativa, entendiendo que a través de ella se producen datos descriptivos (Taylor y Bogdan, 1994: 20) que pueden ayudarnos a alcanzar e interpretar los objetos de una investigación. Por otro lado, como apunta Boase (2013) el creciente número de actividades que se realizan a través de las tecnologías de la información ofrecen una gran oportunidad para la investigación social. Sin embargo, resulta necesario recordar su carácter cambiante y voluble, lo que dificulta las generalizaciones.

En concreto se realizan entrevistas en profundidad semiestructuradas $(n=22)$ a niños $(n=11)$ y niñas $(n=11)$ de entre 9 y 11 años de Zaragoza $(n=11)$ y Pau $(n=11)$. En ambos casos se partió de la elección de individuos de forma aleatoria, con los que no se tenía una relación previa. Para ello se utilizaron contactos indirectos sin que existiera un parámetro fijo para la elección de los entrevistados (situación socioeconómica, procedencia, etc.).

De hecho, la mayor parte de entrevistados eran originarios de la misma localidad (Zaragoza y Pau). La única excepción fue una de las niñas entrevistadas en Zaragoza procede de una familia migrante de Rumanía, si bien se encontraba escolarizada en la capital aragonesa en el momento de la investigación.

No obstante, dada la naturaleza de la investigación, que se limita a la relación de los preadolescentes con la tecnología y, en concreto, el papel que juegan las tablets 
en ella, no se tuvo en consideración este tipo de datos, dado que no formaban parte del objeto de la misma.

En todos los casos los niños entrevistados poseían una tablet, bien en exclusiva, bien compartida con hermanos o padres, independientemente de que algunos de ellos (en ocasiones en edades bien tempranas, como nueve años) también dispusieran de teléfono móvil.

Las entrevistas en profundidad analizaron diversos aspectos relacionados con la tecnología, a través de cuatro grandes apartados:

1. Los espacios donde los preadolescentes realizan sus actividades; 2 . La vigilancia y control por parte de sus padres y la forma en la que escapan de ellos; 3 . Los problemas que a causa del control o vigilancia parental se hayan podido suscitar; $y$ 4. La destreza o dominio de las tecnologías de los jóvenes y de los adultos de sus respectivas familias. En cada uno de los apartados nos interesamos por el significado que los niños otorgan a los usos de la tecnología de la comunicación, especialmente a la tablet.

Estos cuatro apartados se desarrollan en el análisis de resultados a través de tres aspectos concretos desarrollados en las entrevistas y que a su vez se relacionan con las hipótesis planteadas: 1. La posesión de las tablets. 2. Las destrezas digitales y 3. Tecnología y espacio público.

Las entrevistas se llevaron a cabo a lo largo del año 2016 tanto en Zaragoza como en Pau, siendo en Zaragoza realizadas a chicos y chicas del ámbito urbano (Zaragoza ciudad) y en Pau tanto en el ámbito urbano (centre ville de Pau) como en otras poblaciones del área urbana de la localidad (pertenecientes al conocido como agglo de Pau).

\section{Análisis de resultados}

\subsection{La posesión}

Las tablets son elementos tecnológicos plenamente integrados en la vida de los preadolescentes investigados, en Zaragoza y Pau. En todos los casos disponen de una tablet, ya sea propia, ya sea de sus padres/hermanos y están familiarizados con ella. La forma de acceso a la posesión resulta dispar, dependiendo de cada caso, si bien es un asunto común el hecho de que aparecen diferentes familiares a la hora de adquirir o regalar las tablets.

"Era 2015, en Navidad, y mi padrino me dijo que si no comía bien no tendría mi regalo. Me esforcé y él me dio el regalo (tablet). Así fue (...). No fueron mis padres los que la compraron, sino mi padrino, ni mi madre ni mi padre dijeron nada" (Nelly, 11 años, Pau). 
"Me la regalaron mi tía y mi prima, para mi cumpleaños, hace muchos años" (Mario, 9 años, Zaragoza).

A la hora de adquirir o demandar una tablet, en el caso de que no hubiera una previamente (por ejemplo, la de un familiar, padre, madre o hermano mayor), interviene la presión social (Lahire, 2006: 499), la fuerza del grupo de pares, que ya la poseen, que en cierto modo obliga al joven a conseguir este elemento tecnológico.

"No sé muy bien... yo tenía diez años ya, todos mis amigos tenían tablets, así se la pedí a mi madre y, luego, seis meses después, ella habló con mis abuelos y en Navidad tuve mi tablet, con mi primo, que era un año más joven que yo y quería una cámara de fotos" (Malo, 11 años, Pau).

Se trata de un relato muy común en todos, tanto en jóvenes de Zaragoza como de Pau, la necesidad de obtener una tablet porque sus pares (compañeros de clase o de actividades extraescolares) la poseen.

La necesidad de tener el dispositivo crece conforme aumenta la edad del entrevistado. Del mismo modo, la dependencia o necesidad de utilizarla también aumenta con la edad y con la utilización más continuada de los elementos tecnológicos. Algunos de los entrevistados, de menor edad, todavía no eran conscientes de necesitarla o de utilizarla muy habitualmente.

La presencia de un smartphone propio no supone dejar de utilizar la tablet, para la realización de algunas actividades (especialmente juegos, vídeos...). En este sentido, la tablet supone una primera experiencia para desarrollar la personalidad del joven a través de la dimensión digital, que posteriormente continuará con el teléfono.

\subsection{Las destrezas digitales}

Los datos indican que la brecha digital intergeneracional tiende a reducirse, al menos en cuanto a posesión de elementos tecnológicos (Fundación Telefónica, 2018: 102). Entre los preadolescentes investigados la imagen sobre la forma en la que se dominan los dispositivos tecnológicos varía. Por un lado, se encuentran los que consideran que sus conocimientos son superiores a los de sus progenitores. Su visión, por lo tanto, responde al imaginario que concibe a los jóvenes como poseedores de un saber oculto, ajeno a los conocimientos petrificados en las instituciones del saber (Susca, 2012: 121). Una característica, que los padres aprendan también de los hijos, a la que ya se refirió Mead (1970: 1) a mediados del siglo pasado en lo que denominó "culturas prefigurativas", que consideraba que comenzaban a surgir. Siguiendo esa línea Jan Bulck," Kathleen Custers y Sara Nelissen. (2016) proponen estudiar la forma en la que los hijos enseñan a los padres, aprovechando el nuevo ecosistema que surge con la popularización de 
smartphones y tablets. De hecho, trabajos como el de Nelissen y Bulck (2017) estudian este mismo proceso entre 187 familias de Flandes.

Este conocimiento, no obstante, se irá construyendo a lo largo de la vida, a través del juego o de las experiencias compartidas con los pares, alejados habitualmente de los canales de formación oficiales (colegio, instituto), a pesar de que las tablets, como se ha indicado, formen parte desde hace años de la educación formal.

"Bueno... El día que mi madre se compró la tablet, sabía yo más que ella, sí... Ella no sabía cerrar las aplicaciones y yo le di dos veces al botón y ya sabía" (Malena, 10 años, Zaragoza).

En otras ocasiones alguno de los padres sí cuenta con habilidades tecnológicas (a la vista de sus hijos), mientras que el otro carece de ellas. Normalmente es la figura paterna la que se asocia al dominio tecnológico en detrimento de la materna. No obstante, también se dan los casos contrarios.

"Mi madre sí, pero mi padre, no. Para mi tablet, mi madre sí, pero eso es todo, porque ella la compró, aprendió y así mi madre se le da bien la tecnología. Mi padre, no. Porque solo toma fotos para su trabajo. Simplemente llama a la gente y escribe mensajes. Más allá de eso no, mi padre no sabe más sobre tecnología" (Gwen, 9 años, Pau).

"Bueno, mi padre es de (una entidad bancaria) y tiene muchas aplicaciones de esas y sabe usarlas. Mi madre a veces tampoco lo utiliza muy bien, por eso siempre se lo arregla mi padre" (Mikel, 9 años, Zaragoza).

"Sí, bueno, a ver, mi padre más pero mi madre tampoco... Mi madre no es tan buena con la tecnología y todo eso y con los ordenadores y la tele... Casi no sabe ni cómo se enciende y entonces me dice 'Emma, ¿Me ayudas?', pero se lo pide más a mi hermano porque yo sé informática, pero tampoco tanto" (Emma G. C., 9 años, Zaragoza).

La relación de los padres con la tecnología plantea diversas situaciones relacionadas con la seguridad y vigilancia de los jóvenes. A ambos lados de la cordillera se dan relatos que asocian la posesión de elementos tecnológicos (en este caso sobre todo smartphones), con la seguridad de los preadolescentes, que acceden a ellos para que sus padres tengan una mayor tranquilidad, independiente de quien haya partido la iniciativa para obtenerlo.

"Yo de pequeño decía que quería tener móvil y eso. Fue iniciativa mía. Estaba en mi cabeza que quería hablar por WhatsApp... Como veía a mis padres siempre hablar. Quería hablar con mis amigos y eso. Como a veces me iba solo al colegio o a casa o si me iba a algunos sitios solo pues mis padres al final, para navidades, me 
compraron uno, y así me mantienen localizado y están más tranquilos" (Hugo, 10 años, Zaragoza).

Se asocia la posesión de un teléfono móvil con la posibilidad de estar localizado y por lo tanto, más seguro o vigilado. Si bien en cuanto a las posibilidades de vigilancia a través de las tecnologías no existe una respuesta clara de los jóvenes. Una demostración de que el conocimiento que se dispone sobre la tecnología depende de las capacidades del usuario, a pesar de que pueda existir la percepción de que los jóvenes se encuentran mucho más relacionados con la tecnología que sus generaciones precedentes.

\subsection{Tecnología y espacio público}

Sin duda uno de los resultados más interesantes y en el que sí se encuentra una gran diferencia entre los relatos de los jóvenes de ambas ciudades es el que tiene ver con los espacios preferidos por los jóvenes. En este caso existe una clara diferencia entre las vivencias de los jóvenes de uno y otro lado de la cordillera pirenaica.

En el caso de los preadolescentes aragoneses, estos por norma general prefieren encontrarse con sus amigos y se sienten más libres en espacios abiertos (parque, calle, pista o campo de fútbol...) mientras que, en el caso de los bearneses, estos optan por lugares cerrados: principalmente la habitación, la casa o incluso el colegio (recreo).

Los chicos y chicas de Pau asocian estar "fuera" o "en la calle" al peligro. Tan solo en un caso un joven francés manifestó su preferencia por encontrarse fuera, sin embargo, eso no le impide tener miedo a la calle por los peligros que esta puede entrañar. Se trata de la única excepción a una percepción del peligro representada en los coches o los desconocidos.

"Prefiero estar fuera. Porque cuando estoy en la casa, mis padres no piden nada, y cuando estoy afuera soy libre. Puedo ir a ver a mi perro, puedo andar en bicicleta, puedo ir a jugar, puedo ir a caminar. Pero ahora, como mi padre está conmigo los miércoles, a veces vamos en bicicleta. Ahora, puedo disfrutarlo también de él. No voy solo a la calle, porque mi calle es peligrosa." (Jean, 10 años, Pau).

El discurso de los peligros de la calle es muy similar en todos los relatos de los preadolescentes de Pau. Curiosamente, el hecho de que existan eventuales peligros en la calle no impide que los jóvenes entrevistados de Zaragoza prefieran estar y encontrarse ahí con sus amigos, asumiendo los riesgos a los que se puedan enfrentar. Si bien para eso existe una suerte de "libertad pactada" o "vigilada" por parte de los padres, que se encuentran habitualmente a una distancia prudencial de los jóvenes. Como se detalla en estudios precedentes de otros países, la falta de vigilancia o de supervisión de los adultos desempeña un importante papel para 
que los adolescentes decidan ocupar o utilizar los espacios públicos, así como también influye en la forma en la que estos se adaptan a dicha supervisión (Slater, Fitzgibbon y Floyd, 2013; Marwick y Boyd, 2014; Ghosh et al., 2018; Lachance, 2019).

"Con mis amigos prefiero encontrarme en el parque, para jugar. Porque así podemos jugar y si quedamos por ejemplo en otro sitio, con en el colegio, ahí solo tenemos un rato (...) Mis padres me dicen que tengo que estar al lado suyo, porque un amigo mío, que también es del colegio, se fue corriendo a jugar al fútbol a la pista de allá y luego uno pasó con la furgoneta y le dijo 'ven, ven'" (Mario, 9 años, Zaragoza).

A pesar de la tecnología sirve como medio de socialización entre jóvenes y adolescentes, en el caso de los jóvenes preadolescentes de Zaragoza parece muy claro el hecho de que estos prefieren participar en juegos offline que online y hacerlo en espacios abiertos. En el caso de los jóvenes de Pau, la tendencia claramente es a preferir lugares cerrados en los que, en muchos casos, sí que interviene la tecnología.

"Me gusta estar en casa de mi amiga, en su jardín o así. En un lugar seguro.

Por ejemplo, en su jardín, estoy segura que está bastante protegido. En la calle nunca sabes con quién te puedes encontrar" (Carole, 11 años, Pau).

"Prefiero estar en casa, porque en mi casa (en mi habitación), me puedo divertir, puedo jugar y puedo hacer lo que quiero" (Nelly, 9 años, Pau).

"(Cuando estoy solo, en casa) juego con mis Lego, estoy con mi tablet o estoy con mi Playstation" (Guillaume, 11 años, Pau).

\section{Discusión y conclusiones}

Entre los resultados obtenidos destaca que, tal y como puede parecer previsible, la forma en la que los preadolescentes de Zaragoza y Pau entrevistados utilizan las tablets resulta muy similar. Tanto por su propia familiarización con este dispositivo como por el tipo de actividades que con él realizan. En este sentido, la utilización de la tablet no se diferencia de las actividades observadas en Estados Unidos u otros países entre los preadolescentes o preteens (Rideout, 2015; Lauricella et al., 2014; OFCOM, 2014; Beyens y Beullens, 2017).

La tablet representa un primer contacto con el mundo digital, en muchos casos previo a la adquisición del smartphone, un elemento que supone el comienzo de la madurez digital, el paso al mundo adulto. A su vez su posesión y uso entre los preadolescentes de Zaragoza y Pau se encuentra totalmente generalizado, por lo tanto, se confirma la primera de las hipótesis planteadas: (H1). 
Al mismo tiempo, observada la penetración de estos elementos tecnológicos y el papel que juegan en las vidas de los preadolescentes entrevistados, se confirma también que el nivel de penetración tecnológica a ambos lados del Pirineo resulta muy similar. (H2).

Se trata de un hecho que puede parecer provocado por la generalización de las tecnologías de la información en todo el mundo y que afecta especialmente a Europa Occidental, como una de las regiones del planeta donde mayores cuotas ha alcanzado esta penetración tecnológica. A diferencia de lo que ocurrió en el pasado, cuando los adelantos llegaron de un modo mucho más lento o escalonado, la penetración tecnológica llega a todas las partes, de un modo más uniforme.

Las principales diferencias observadas en la presente investigación radican en el significado que se otorga a las tablets en relación con otras actividades que desarrollan los preadolescentes de uno y otro lado del Pirineo. Se observa que mientras en Zaragoza los jóvenes prefieren encontrarse con sus pares en lugares abiertos, preferentemente en la calle, donde poder socializarse, en Pau la tendencia es a buscar ese mismo lugar en espacios cerrados (habitación, casa, escuela...).

Esta observación llama la atención sobre la posibilidad de que pueda darse una explicación cultural a este fenómeno, que en la cultura ibérica exista una tendencia a vivir y socializarse en la calle frente a la continental, más recluida. Pero sorprenden las referencias al miedo, presente sobre todo en los testimonios de los preadolescentes franceses. Los datos sobre criminalidad en ambos países indican que Francia registra mayores niveles de delincuencia que España (ver Anexo 3), pero resulta discutible que estos datos se hayan interiorizado en la población de tal forma hasta el punto de generar un imaginario tan poderoso incluso entre sus generaciones más jóvenes. Como se refleja en algunos estudios (Smith et al., 2015; Hecke et al., 2016) son múltiples los factores que hacen o no atractivos los espacios públicos abiertos entre los adolescentes. Por lo tanto, puede resultar esta una interesante cuestión a plantear en posteriores investigaciones y que queda fuera de los límites del presente trabajo.

Como consecuencia, se descarta, al menos parcialmente, la tercera de las hipótesis $(\mathrm{H} 3)$, demostrándose una clara diferencia entre los preadolescentes de las dos ciudades analizadas. Si bien en el caso de Zaragoza la hipótesis se cumple, en el caso de Pau se observa cómo el espacio público no supone un lugar preferido para la socialización de los preadolescentes.

Por lo tanto, no es el uso de la tablet el que cambia de una población a otra, sino el hecho de preferir espacios abiertos o cerrados para conocer y socializarse con sus pares. De estas elecciones se puede interpretar que una u otra decisión (encontrarse en un lugar abierto -público- o cerrado -privado-) puede desempeñar un papel en el aumento o reducción de situaciones favorables para usar este aparato. La tablet es una herramienta digital utilizada principalmente en espacios 
cerrados y de forma menos habitual en espacios abiertos. En otras palabras, es posible que no sea la relación con el espacio la que transforme el uso de la herramienta digital, sino que el tipo de ocupación que se otorga al espacio el que induce a usos específicos de la tablet. Un análisis que puede resultar importante explorar en futuras investigaciones, para conocer la forma en la que nos relacionamos a través de la tecnología, así como el papel que desempeñan dispositivos como las tablets, especialmente en determinados lugares y colectivos. 


\section{Referencias bibliográficas}

ASOREY, E. y GIL, J. (2009): El placer de usar las TIC en el aula de Infantil. CEE Participación Educativa, $n^{\circ}$ 12. Madrid, Ministerio de Consejo Escolar del Estado, pp. 110-119. Disponible en:

https://sede.educacion.gob.es/publiventa/descarga.action?f_codigo_agc=14009_ 19

BEYENS, I. y BEULLENS, K. (2017): Parent-child conflict about children's tablet use: The role of parental mediation. New Media \& Society, v. 19, $n^{\circ} 12$. Thousand Oaks, SAGE Journals, pp. 2075-2093. doi: 10.1177/1461444816655099

BOASE, J. (2013): Implications of software-based mobile media for social research. Mobile Media \& Communication, vol. 1, $\mathrm{n}^{\circ}$ 1. Thousand Oaks, SAGE Journals, pp. 57-62. doi: 10.1177/2050157912459500

BOYD, D. (2008): Why Youth (Heart) Social Network Sites: The Role of Networked Publics in Teenage Social Life. En Buckingham, D. (Ed.), Youth, Identity and Digital Media. Cambridge, MIT Press, pp. 119-142. doi: 10.1162/dmal.9780262524834.119.

BULCK, J., CUSTERS, K. y NELISSEN, S. (2016): The child-effect in the new media environment: Challenges and opportunities for communication research. Journal of Children and Media, vol. 10, $\mathrm{n}^{\circ}$ 1. Abingdon, Taylor \& Francis, pp. 30-38. doi: 10.1080/17482798.2015.1121897

CÁCERES, M. P., HINOJO, F. J. y AZNAR, I. (2011): Incorporación de las TIC en el período escolar de 0 a 6 años: diseño de una entrevista para evaluar las percepciones de los maestros. Píxel-Bit. Revista de Medios y Educación, n 39. Sevilla, Universidad de Sevilla, pp. 7-16. Disponible en: https://recyt.fecyt.es/index.php/pixel/article/view/61446/37459

CAHILL, C. (2000): Street literacy: Urban teenagers' strategies for negotiating their neighbourhood. Journal of Youth Studies, vol. 3, $n^{\circ} 3$. Abingdon, Taylor \& Francis, pp. 251-277. doi: 10.1080/713684375.

CAUWENBERGE, A., D'HAENENS, L. y BEENTJES, H. (2015): How to take advantage of tablet computers: Effects of news structure on recall and comprehension. Communications: The European Journal of Communication Research, vol. 40, n 4. Berlín, De Gruyter, pp. 425-446. doi: 10.1515/commun2015-0020

CHAN-OLMSTED, S. y SHAY, R. (2016): Understanding tablet consumers: Exploring the factors that affect tablet and dual mobile device ownership. Journalism and 
Mass Communication Quarterly, vol. 93, $n^{\circ} 4$. Thousand Oaks, SAGE Journals, pp. 857-883. doi: 10.1177/1077699016644561

CHYI, H. I. y CHADHA, M. (2012): News on new devices: Is multi-platform news consumption a reality? Journalism Practice, vol. $6, \mathrm{n}^{\circ} 4$. Abingdon, Taylor \& Francis, pp. 431-449. doi: 10.1080/17512786.2011.629125

CONNELL, S. L., LAURICELLA, A. R., y WARTELLA, E. (2015): Parental co-use of media technology with their young children in the USA. Journal of Children and Media, vol 9, n 1. Abingdon, Taylor \& Francis, pp. 5-21.

doi: 10.1080/17482798.2015.997440

CREDOC (2016): Le baromètre du numérique 2016. París, Ministère de l'économie et des finances. Disponible en: https://www.credoc.fr/publications/barometre-dunumerique-edition-2016

DANBY, S., DAVIDSON, C., THEOBALD, M., SCRIVEN, B., COBB-MOORE, C., HOUEN, S., GRANT, S., GIVEN, L. M. y THORPE, K. (2013): Talk in activity during young children's use of digital technologies at home. Australian Journal of Communication, vol. 40, $\mathrm{n}^{\circ}$ 2. Brisbanem, Communication Institute, pp. 83-99. Disponible en:

https://www.academia.edu/17971767/Talk_in_activity_during_young_children_s_u se_of_digital_technologies_at_home

DEBORD, G. (2005): La sociedad del espectáculo. Valencia: Pre-textos. Segunda reimpresión (segunda edición).

D'HEER, E. y COURTOIS, C. (2014): The changing dynamics of television consumption in the multimedia living room. Convergence, vol. $22, n^{\circ} 1$. Thousand Oaks, SAGE Journals, pp. 3-17. doi: 10.1177/1354856514543451

DIAS, P. (2016): Motivations for multi-screening: An exploratory study on motivations and gratifications. European Journal of Communication, vol. 31, $n^{\circ} 6$. Thousand Oaks, SAGE Journals, pp.678-693. doi: 10.1177/0267323116674111

DITENDRIA (2018): Informe Ditendria: Mobile en España y en el mundo en 2018. Madrid, Ditendria. Disponible en: https://ditrendia.es/informe-mobile-2018/

DUNAWAY, J., SEARLES, K., SUI, M., y NEWLY, P. (2018): News attention in a mobile era. Journal of Computer-Mediated Communication, vol. 23, $n^{\circ} 2$. Oxford, Oxford University Press, pp. 107-124. doi: 10.1093/jcmc/zmy004

DUURSMA, E., MEIJER, A. y DE-BOT, K. (2017): The Impact of Home Literacy and Family Factors on Screen Media Use among Dutch Preteens. Journal of Child and 
Family Studies, vol. 26, $\mathrm{n}^{\circ}$ 2. Heidelberg, Springer Science, pp. 612-622. doi: $10.1007 / \mathrm{s} 10826-016-0584-5$

FEIXA, C. (2014): De la Generación@ a la \#Generación. La juventud en la era digital. Barcelona: NED Ediciones.

FERNÁNDEZ, E. (1997): Gente de orden. Tomo 3. La economía. Zaragoza: Ibercaja.

FIDLER, R. (1998): Mediamorfosis. Comprender los nuevos medios. Buenos Aires: Ediciones Juan Granica.

FIGUERAS-MAZ, M, MASANET, M, y FERRÉS, J. (2017): Mobile devices in higher education: A pending issue in multidimensional media literacy. Catalan Journal of Communication \& Cultural Studies, vol. 9, n 1 . Bristol, Intellect, pp. 135-144. doi: $10.1386 /$ cjcs.9.1.1351

FLUCKIGER, C. (2006): La sociabilité juvénile instrumentée. L'appropriation des blogs dans un groupe de collégiens. Réseaux, vol. 4, n 138. París, La Découverte, pp. 111-138. doi: 10.3917/res.138.0109.

FUNDACIÓN TELEFÓNICA (2017): Sociedad Digital en España 2017. Sdi[17. Madrid-Barcelona: Fundación Telefónica-Editorial Ariel.

(2018): Sociedad Digital en España 2016. Sdi[16. Madrid-Barcelona: Fundación Telefónica-Editorial Ariel.

GERMÁN, L. (2012): Historia económica de Aragón contemporáneo. Zaragoza: Consejo Económico y Social de Aragón (CESA).

GERSHON, R. A. (2013): Digital media innovation and the Apple iPad: Three perspectives on the future of computer tablets and news delivery. Journal of Media Business Studies, vol. 10, $\mathrm{n}^{\circ}$ 1. Abingdon, Taylor \& Francis, pp. 41-62. doi: 10.1080/16522354.2013.11073559

GHOSH, A. K., BADILLO-URQUIOLA, K., GUHA, S., LAVIOLA, J. J. y WISNIEWSKI, P. J. (2018): Safety vs. Surveillance: What Children Have to Say about Mobile Apps for Parental Control. CHI '18: Proceedings of the $2018 \mathrm{CHI}$ Conference on Human Factors in Computing Systems, 14. Montreal, Association of Computing Machinery, pp. 1-14. doi: 10.1145/3173574.3173698

GIL, H., y LIU, J. H. (2017): Second screening politics in the social media sphere: Advancing research on dual screen use in political communication with evidence from 20 countries. Journal of Broadcasting and Electronic Media, vol. 61, $\mathrm{n}^{\circ} 2$. Abingdon, Taylor \& Francis, pp. 193-219. doi: 10.1080/08838151.2017.1309420 
GLÉVAREC, H. (2010): Les trois âges de la 'culture de la chambre'. Ethnologie française, vol. 140, $n^{\circ} 1$. París, Presses Universitaires de France, pp. 19-30. doi: 10.3917/ethn.101.0019.

HECKE, L., DEFORCHE, B., DYCK, D., BOURDEADHUIJ, I., VEITCH, J. y CAUWENBERG, J. (2016): Social and Physical Environmental Factors Influencing Adolescents' Physical Activity in Urban Public Open Spaces: A Qualitative Study Using Walk-Alone Interviews. Health \& Place, Vol. 11, $n^{\circ}$ 5. Amsterdam, Elsevier, pp. 158-173. doi: 10.1371/journal.pone.0155686

HERNÁNDEZ, K. D., YÁÑEZ, J. F. y CARRERA, A. A. (2017): Las redes sociales y adolescencias. Repercusión en la actividad física. Revista Universidad y Sociedad, vol. 9, $\mathrm{n}^{\circ}$ 2. Cienfuegos, Universo Sur, pp. 242-247. Disponible en: http://scielo.sld.cu/scielo.php?script=sci_arttext\&pid=S2218-36202017000200033

HOVE, S., VANDERHOVEN, E. y CORNILLIE, F. (2017): La tablet para el aprendizaje de vocabulario en segundas lenguas: teclado, lápiz digital u opción múltiple. Revista Comunicar. Revista científica iberoamericana de comunicación y educación, $n^{\circ}$ 50. Huelva, Grupo Comunicar, pp. 53-63. doi: 10.3916/C50-2017-05

INSTITUT NATIONAL DE STATISTIQUE ET DES ÉTUDES ÉCONOMIQUES (INSEE) (2017): Tableaux de l'économie française. Disponible en: https://www.insee.fr/fr/statistiques/2587886 [Consultado: 18 de octubre de 2018]

INSTITUTO NACIONAL DE ESTADÍSTICA (INE) (2017): Encuesta sobre Equipamiento y Uso de Tecnologías de Información y Comunicación en los hogares 2017. Disponible en:

https://www.ine.es/dyngs/INEbase/es/operacion.htm?c=Estadistica_C\&cid=12547 $36176741 \&$ menu=ultiDatos\&idp=1254735976608 [Consultado:12 de octubre de 2018]

(2019a): Contabilidad Regional de España. Resultados por comunidades y ciudades autónomas. Disponible en:

https://www.ine.es/dyngs/INEbase/es/operacion.htm?c=Estadistica_C\&cid=12547 36167628\&menu=resultados\&idp $=1254735576581 \#$ !tabs-1254736158133

(2019b): Cifras oficiales de población resultantes de la revisión del Padrón municipal a 1 de enero. Disponible en:

https://www.ine.es/jaxiT3/Datos.htm?t=2853\#!tabs-tabla

LACHANCE, J. (2011): L'adolescence hypermoderne. Le nouveau rapport au temps des jeunes. Québec: Presses de l'Université Laval.

(2013): Photos d'ados à l'ère du numérique. Québec: Presses de I'Université Laval. 
(2016): Le smartphone: unZobjet?transitionnel ou interactionne/? L'école des Parents, vol. 6, n 621. París, Eres, pp. 105-114. doi: 10.3917/epar.s621.0105

(2019): Parental surveillance of teens in the digital era: the 'ritual of confession' to the 'ritual of repentance'. International Journal of Adolescence and Youth, vol. $25, n^{\circ} 1$. Abingdon, Taylor \& Francis, pp. 353-363.

doi: 10.1080/02673843.2019.1651351

LAHIRE, B. (2006): La culture des individus. Dissonances culturelles et distinction de soi. París: La Découverte/Poche.

LAURICELLA, A. R., CINGEL, D. P., BLACKWELL, C., WARTELLA, E. y CONWAY, A. (2014): The mobile generation: Youth and adolescent ownership and use of new media. Communication Research Reports, vol. $31, n^{\circ} 4$. Abingdon, Taylor \& Francis, pp. 357-364. doi: 10.1080/08824096.2014.963221

LÉVY, P. (2004): Inteligencia colectiva. Por una antropología del ciberespacio. Washington D. C.: Bilbioteca Virtual em Saúde. Disponible en: http://inteligenciacolectiva.bvsalud.org/public/documents/pdf/es/inteligenciaCole ctiva.pdf

LIVINGSTON, S. (2007): From family television to bedroom culture: Young people's media at home. En Devereux, E. (ed.), Media Studies: Key issues and Debates. London, Sage Publications, pp. 302-321. Disponible en:

http://eprints.Ise.ac.uk/2772/1/From_family_television_to_bedroom_culture_(LSER O).pdf

LOMBORG, S. y MORTENSEN, M. (2017): Users across media: An introduction. Convergence, vol. 23, $n^{\circ}$ 4. Thousand Oaks, SAGE Journals, pp. 343-351. doi: $10.1177 / 1354856517700555$

MACKAY, J. e IVORY, A. (2014): Mobile devices and transforming journalistic routines. The Electronic Journal of Communication, vol. 24, n 3-4. Rotterdam Junction, Communication Institute for Online Scholarships. Disponible en: http://www.cios.org/EJCPUBLIC/024/3/024342.html

MADISON, E. (2015): Mobile media best practices: Lessons from 5 years of OR magazine. Journalism \& Mass Communication Educator, vol 70, $n^{\circ} 3$. Thousand Oaks, SAGE Journals, pp. 324-330. doi: 10.1177/1077695815600479

MANOVICH, L. (2005): El lenguaje de los nuevos medios de comunicación. La imagen en la era digital. Barcelona: Paidós Comunicación. 
MARWICK, A. E. y BOYD, D. (2014): Networked privacy: How teenagers negotiate context in social media. New Media \& Society, vol. 16, $n^{\circ} 7$. Thousand Oaks, SAGE Journals, pp. 1051-1067. doi: 10.1177/1461444814543995

MCCREERY, S. P. y KRUGMAN, D. M. (2015): TV and the iPad: How the tablet is redefining the way we watch. Journal of Broadcasting and Electronic Media, vol. 59, $n^{\circ} 4$. Abingdon, Taylor \& Francis, pp. 620-639.

doi: 10.1080/08838151.2015.1093483

MCLUHAN, M. (1996): Comprender los medios de comunicación. Las extensiones del ser humano. Barcelona: Paidós.

MEAD, M. (1970): Culture and commitment. A study of the Generation Gap. New York: Natural History Press.

MILLER, J. L. y KOCUREK, C. A. (2017): Principles for educational game development for young children. Journal of Children and Media, vol. 11, $n^{\circ} 3$. Abingdon, Taylor \& Francis, pp. 314-329. doi: 10.1080/17482798.2017.1308398

MORRIS, J. y PATTERSON, E. (2015): Podcasting and its apps: Software, sound, and the interfaces of digital audio. Journal of Radio \& Audio Media, vol. 22, $\mathrm{n}^{\circ} 2$. Abingdon, Taylor \& Francis, pp. 220-230. doi: 10.1080/19376529.2015.1083374

MYRTIL, M. J., JUSTICE, L. M., PELFREY, L., LOGAN, J. A., XIE, K. y BARNES, L. (2018): Preschool Teachers' Implementation Fidelity When Using a TechnologyMediated Language and Literacy Intervention. Child \& Youth Care Forum, vol. 47, $n^{\circ}$ 6. Heidelberg, Springer Science, pp. 771-786. doi: 10.1007/s10566-018-9460-3

NEIJENS, P. C., y VOORVELD, H. A. M. (2018): Digital replica editions versus printed newspapers: Different reading styles? Different recall? New Media \& Society, vol. 20, $n^{\circ}$ 2. Thousand Oaks, SAGE Journals, pp. 760-776. doi: $10.1177 / 1461444816670326$

NELISSEN, S. y BULCK, J. (2017): When digital natives instruct digital immigrants: Active guidance of parental media use by children and conflict in the family. Information, Communication \& Society, vol. 21, $n^{\circ} 3$. Abingdon, Taylor \& Francis, pp. 375-387. doi: 10.1080/1369118X.2017.1281993

NEUMANN, M. M. (2017): Parent scaffolding of young children's use of touch screen tablets. Early Child Development and Care, vol. 188, $n^{\circ} 12$. Abingdon, Taylor \& Francis, pp. 1654-1664. doi: 10.1080/03004430.2016.1278215

NOGUEIRA, M. A. y CEINOS, C. (2015): Influencia de la Tablet en el desarrollo infantil: Perspectivas y recomendaciones a tener en cuenta en la orientación familiar. 
Técnicas pedagógicas, $n^{\circ}$ 26. Madrid, Universidad Autónoma de Madrid, pp. 3350. Disponible en:

https://revistas.uam.es/tendenciaspedagogicas/article/view/2120/2216

O'CONNOR, J. R. y JACKSON, K. N. (2016): The Use of iPad® Devices and 'Apps' for ASD Students in Special Education and Speech Therapy. En KATS, Y. (ed.): Supporting the Education of Children with Autism Spectrum Disorders. Hershey, IGI Global, pp. 267-283. doi: 10.4018/978-1-5225-0816-8.ch014

OFCOM (2014): Children and parents: media use and attitudes report. London, Ofcom. Disponible en:

https://www.ofcom.org.uk/research-and-data/media-literacyresearch/childrens/children-parents-oct-14

ONTSI (2019): La sociedad en Red. Transformación digital en España. Informe Anual 2018. Edición 2019. Madrid: ONTSI. doi: 10.30923/1989-7424-2019

ORTIZ, A., PRATS, M. y BAYLINA, M. (2014): Procesos de apropiación adolescente del espacio público: otra cara de la renovación urbanística en Barcelona. Boletín de la Asociación de Geógrafos Españoles, n 65: Madrid, Asociación Española de Geografía, pp. 37-57. doi: 10.21138/bage.1742

PHAM, B. y LIM, S. S. (2019): Vietnamese pre-schoolers' tablet use and early childhood learning: An ecological investigation. Journal of Children and Media, vol. 13, $\mathrm{n}^{\circ}$ 3. Abingdon, Taylor \& Francis, pp. 241-259. doi: 10.1080/17482798.2019.1613247

PISANI, F. y PIOTET, D. (2009): La alquimia de las multitudes. Cómo la web está cambiando el mundo. Barcelona: Paidós.

RAMOS, R. (2016): Penetración tecnológica en Aragón. De periferia a centro. Revista F@ro, vol. 1, n²3. Valparaíso, Universidad de Playa Ancha. Facultad de Ciencias Sociales, pp. 143-167. Disponible en:

https://www.revistafaro.cl/index.php/Faro/article/view/463/409

REINA, E., PÉREZ, R. y QUERO, N. (2017): Utilización de tablets en Educación Infantil: Un estudio de caso. Revista Latinoamericana de Tecnología Educativa, vol. 16, $n^{\circ}$ 2. Badajoz, Departamento de Ciencias de la Educación. Universidad de Extremadura, pp. 193-203. doi: 10.17398/1695-288X.16.2.193.

RIDEOUT, V. (2015): The common sense census: Media use by tweens and teens. United States of America, Common Sense. Disponible en:

https://www.commonsensemedia.org/sites/default/files/uploads/research/census_ researchreport.pdf 
(2016): Measuring time spent with media: The Common Sense census of media use by US 8- to 18-year-olds. Journal of Children and Media, vol. 10, $\mathrm{n}^{\circ} 1$. Abingdon, Taylor \& Francis, pp. 138-144. doi: 10.1080/17482798.2016.1129808

SÁNCHEZ, C. y RICOY, M. (2018): Posicionamiento de la familia ante el uso de la tableta en el aprendizaje del alumnado de Educación Primaria. Digital Education Review, $\mathrm{n}^{\circ}$ 33. Barcelona, Universitat de Barcelona. Digital Education Observatory (OED), pp. 267-283. doi: 10.1344/der.2018.33.267-283

SCHWARZ, O. (2009): Good Young Nostalgia: Camera Phones and Technologies of Self among Israeli Youths. Journal of Consumer Culture, vol. 9, $n^{\circ} 3$. Thousand Oaks, SAGE Journals, pp. 348-376. doi: 10.1177/1469540509342045

SHUTER, R., CHEONG, P. y CHEN, Y. (2016): The influence of cultural values on U.S. and Danish students' digital behavior: Exploring culture, new media, and social context. Journal of International and Intercultural Communication, vol. 9, $\mathrm{n}^{\circ} 2$. Abingdon, Taylor \& Francis, pp. 161-178. doi: 10.1080/17513057.2016.1154183

SLATER, S., FITZGIBBON, M. y FLOYD, M. F. (2013): Urban Adolescent's Perceptions of their Neighborhood Physical Activity Environments. Leisure Sciences. An interdisciplinary Journal, vol. 35, $n^{\circ}$ 2. Abingdon, Taylor \& Francis, pp. 167-183. doi: 10.1080/01490400.2013.761912

SMITH, A. L., TROPED, P. J., McDONOUGH, M. H. y DEFREESE, J. D. (2015): Youth perceptions of how neighborhood physical environment and peers affects physical activity: a focus group. International Journal of Behavioral Nutrition and Physical Activity, $\mathrm{n}^{\circ}$ 12. Minneapolis, International Society of Behavioral Nutrition and Physical Activity, artículo n 80. doi: 10.1186/s12966-015-0246-9

SOYKAN, E. (2015): Views of students', teachers' and parents' on the tablet computer usage in education. Cypriot Journal of Educational Sciences, vol. 10, $\mathrm{n}^{\circ}$ 3. Nicosia, Department of Computer Education and Instructional Technology. Near East University, pp. 228-244. doi: 10.18844/cjes.v1i1.68

SUSCA, V. (2012): Deleite trágico. Las formas elementales de la vida electrónica. Barcelona: Península.

TAYLOR, S. J. y BOGDAN, R. (1994): Introducción a los métodos cualitativos de investigación. La búsqueda de significados. Barcelona: Paidós. Segunda reimpresión

VIDALES-BOLAÑOS, M. J. y SÁDABA-CHALEZQUER, C. (2017): Adolescentes conectados: La medición del impacto del móvil en las relaciones sociales desde el capital social. Revista Comunicar. Revista científica iberoamericana de 
comunicación y educación, $n^{\circ}$ 53. Huelva, Grupo Comunicar, pp. 19-28. doi: 10.3916/C53-2017-02

WARTELLA, E., RIDEOUT, V., LAURICELLA, A. R. y CONNELL, S. A. (2014): Parenting in the Age of Digital Technology. A National Survey. Evanston, Northwestern University. Disponible en: https://cmhd.northwestern.edu/wpcontent/uploads/2015/06/ParentingAgeDigitalTechnology.REVISED.FINAL_.2014. pdf

WEI, R. (2013): Mobile media: Coming of age with a big splash. Mobile Media \& Communication, vol. 1, $\mathrm{n}^{\circ}$ 1. Thousand Oaks, SAGE Journals, pp. 50-56. doi: $10.1177 / 2050157912459494$

WILLEM, C., TORTAJADA, I. y FIGUERAS-MAZ, M. (2017): Teens just wanna have fun? Adolescents' construction of desirability on SNS in Spain. Comunicació. Revista de recerca i d'Anàlisi [Societat Catalana de Comunicació], vol. 34, n 2. Barcelona, Societat Catalana de Comunicació, pp. 77-92. doi: 10.2436/20.3008.01.161

ZHU, S., YANG, H. H., McLEOD, J., SHI, Y. y WU, D. (2018). Parent's and Student's attitudes toward tablet integration in Schools. International Review of Research in Open and Distributed Learning. vol. 19, N ${ }^{\circ}$. Athabasca, Athabasca University, 222 241. doi: 10.19173/irrodl.v19i4.2970 


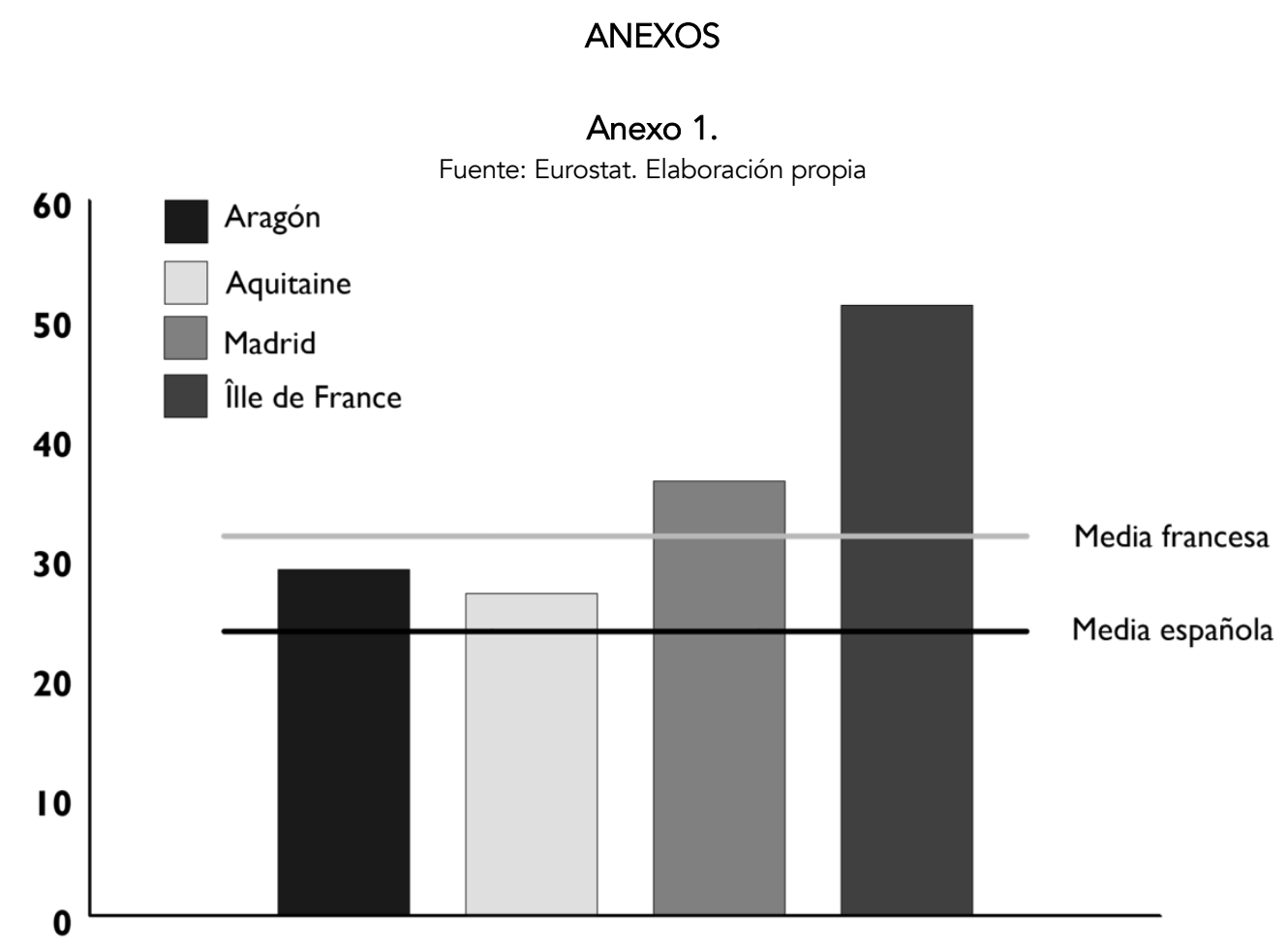

Gráfico 1. Gráfico comparativo del PIB per cápita en Aragón, Aquitaine, Madrid e îlle de France. Datos en miles de euros, correspondientes a 2016.

Anexo 2.

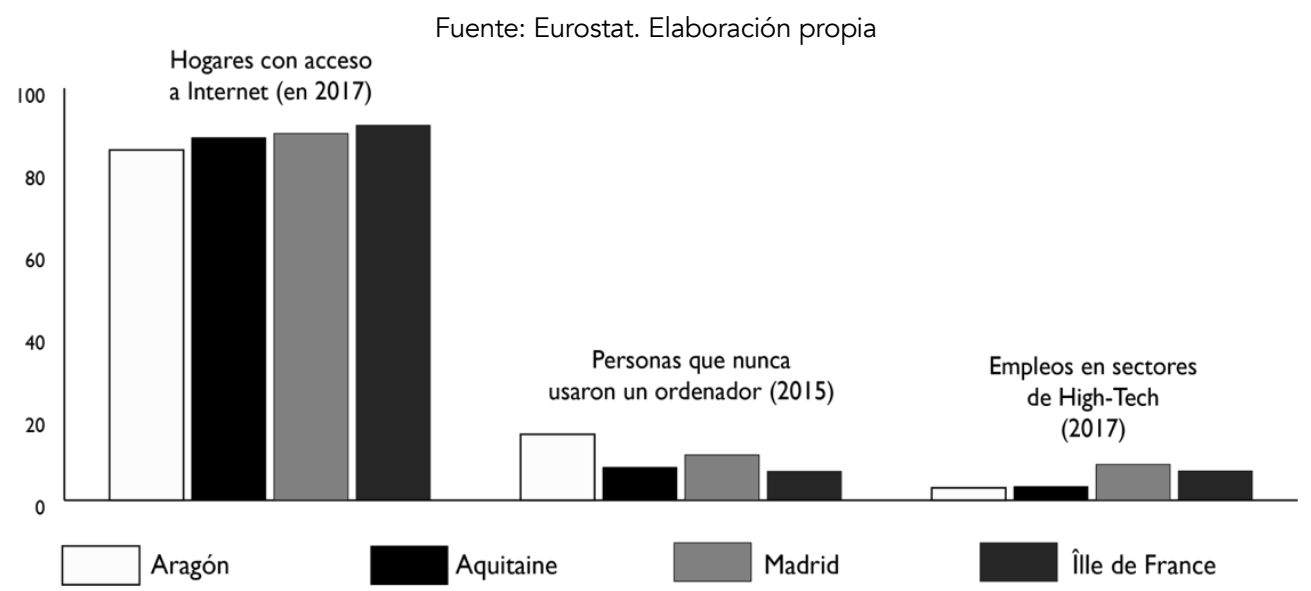

Gráfico 2. Gráfico comparativo de datos sobre la penetración tecnológica en Aragón, Aquitaine, Madrid e îlle de France 


\section{Anexo 3.}

Tabla 1. Comparación entre los principales índices de delincuencia en España y Francia. Delitos por cada 100.000 habitantes en España y Francia (2015)

\begin{tabular}{|l|l|l|}
\hline Tipo de Delito & España & Francia \\
\hline Asalto & 62,99 & 379,12 \\
\hline Rapto & 0,20 & 6,18 \\
\hline Robo sin violencia & 446,10 & 1906,92 \\
\hline Robo con violencia & 140,02 & 162,92 \\
\hline Homicidios & 0,65 & 1,57 \\
\hline Total Violencia sexual & 21,39 & 52,06 \\
\hline
\end{tabular}

Fuente: United Nations Office on Drugs and Crime 